\title{
Epstein-Barr virus-associated smooth muscle tumor of the cavernous sinus: a delayed complication of allogenic peripheral blood stem cell transplantation: case report
}

\author{
Amol Raheja, MBBS, ${ }^{1}$ Aleksandra Sowder, MD, ${ }^{2}$ Cheryl Palmer, MD, ${ }^{2}$ Fausto J. Rodriguez, MD, ${ }^{3}$ \\ and William T. Couldwell, MD, PhD'
}

\begin{abstract}
'Department of Neurosurgery, Clinical Neurosciences Center, and 'Department of Pathology, University of Utah, Salt Lake City, Utah; and ${ }^{3}$ Department of Pathology, The Johns Hopkins Hospital, Baltimore, Maryland
\end{abstract}

\begin{abstract}
Epstein-Barr virus (EBV)-associated smooth muscle tumors (SMTs) have recently been associated with primary and secondary immunodeficiencies. They are broadly divided into 3 subgroups: HIV-related, posttransplant, and congenital immunodeficiency. Subsequent to organ transplantation and acquired immunosuppression, a few cases of EBV-associated SMTs have been described in the liver, respiratory tract, and gastrointestinal system. To the authors' knowledge, intracranial involvement after peripheral blood stem cell transplantation has never been reported previously. The authors describe the case of a 65 -year-old woman who presented with recent-onset painful ophthalmoplegia. She had a prior history of acute myelogenous leukemia requiring allogenic peripheral blood stem cell transplantation 2 years earlier, but she was in a remission phase. Imaging revealed a T1/T2 isointense, homogeneously enhancing lesion of the left cavernous sinus. A presumptive diagnosis of Tolosa-Hunt syndrome was made, and she was treated with steroids; however, her symptoms progressed quickly and repeat imaging revealed that the lesion was growing. To rule out leukemic deposits, a minimally invasive lateral orbitotomy extradural transcavernous approach was performed for biopsy sampling and debulking of the lesion. The biopsied tumor tissue was found to be infiltrative, grayish, firm, and moderately vascular. The final pathology results indicated an EBV-associated SMT of the cavernous sinus. Subsequently, the patient's steroid treatment was stopped and she had obtained partial symptomatic relief at her last follow-up visit, 3 months after surgery. EBV-associated SMT should be included in the differential diagnosis for intracranial and dural-based central nervous system lesions, especially in immunocompromised patients. Paradoxical response to steroids with worsening of symptoms is a hallmark of EBV-associated SMTs.
\end{abstract}

https://thejns.org/doi/abs/10.3171/2016.4.JNS16290

KEY WORDS cavernous sinus; minimally invasive biopsy; lateral orbitotomy extradural transcavernous approach; EBV-associated smooth muscle tumor; peripheral blood stem cell transplantation; Epstein-Barr virus; oncology

$\mathrm{E}$ PSTEIN-BARR virus (EBV) is a DNA herpes virus that has the potential to immortalize infected cells. ${ }^{3}$ It has traditionally been linked to pathogenesis of Burkitt lymphoma, nasopharyngeal carcinoma, gastric carcinoma, and other B-cell lymphomas in immunocompromised patients. ${ }^{13}$ Suppression of the immune system leads to an increased propensity for development of neoplasia-like EBV-associated smooth muscle tumors (SMTs). ${ }^{7}$ Although this association was first described by Pritzker et al..$^{15}$ in 1970, it was not until 1995 that the EBV genome was first implicated in the pathogenesis of SMTs. ${ }^{12,13}$ Recognition of the association with EBV has streamlined the classification of these SMTs, which were formerly diagnosed as leiomyoma or leiomyosarcomas. ${ }^{7}$

SMTs occur primarily in patients with induced immunosuppression, either posttransplantation (PT-SMT) or associated with HIV, or in those with congenital immunodeficiency; they follow a distinct natural history in each subgroup of patients. ${ }^{7}$ HIV-SMT and PT-SMT have a similar incidence, but congenital immunodeficiency SMT has been described less frequently.? Overall, there is mild female preponderance. The pathophysiology of these subtypes is poorly understood.

Only 7 cases of intracranial EBV-associated PT-SMT

ABBREVIATIONS EBER = EBV-encoded small RNA; EBV = Epstein-Barr virus; ICA = internal carotid artery; LOET = lateral orbitotomy extradural transcavernous; PT-SMT = posttransplantation SMT; SMA = smooth muscle actin; SMT = smooth muscle tumor; THS = Tolosa-Hunt syndrome.

SUBMITTED February 4, 2016. ACCEPTED April 14, 2016.

INCLUDE WHEN CITING Published online June 24, 2016; DOI: 10.3171/2016.4.JNS16290. 
have been documented in the literature so far, all in solidorgan (kidney and lung) transplant recipients., , $^{3,9-11,17,21}$ To the best of our knowledge, we report the first case of intracranial involvement of an EBV-associated SMT after allogenic peripheral blood stem cell transplantation in an adult. The patient initially presented with symptoms consistent with Tolosa-Hunt syndrome (THS), but treatment with steroids led to paradoxical worsening of her clinical symptoms. We used a minimally invasive approach for cavernous sinus lesions - a lateral orbitotomy extradural transcavernous (LOET) approach under stereotactic neuronavigation guidance - to safely obtain a biopsy and debulk the cavernous sinus lesion under direct vision. ${ }^{1}$

\section{Case Report}

\section{Clinical Presentation and Radiological Imaging}

A 65-year-old woman presented to our clinic with recent-onset painful ophthalmoplegia of 6 months' duration. She had a history of acute myelogenous leukemia diagnosed 3 years previously for which she underwent allogenic peripheral blood stem cell transplantation 2 years earlier. She had subsequently undergone multiple cycles of chemotherapy for frequent relapses; however, she was in remission at the time of her current visit with normal cell counts. She was initially seen by an ophthalmologist, who ordered gadolinium-enhanced MRI (Fig. 1A). Images revealed a $6 \times 8 \times 12-\mathrm{mm} \mathrm{T1} / \mathrm{T} 2$ isointense, homogeneously enhancing lesion of the left cavernous sinus with its predominant bulk anterolateral to the internal carotid artery (ICA). There was no evidence of diffusion restriction or hemorrhage in the lesion. Her primary radiological differential diagnoses included meningioma, leukemic deposits, and THS.

Considering the rapid onset and progression of symptoms and remission of systemic disease, a working diagnosis of THS was made, and a course of oral steroids was initiated. Rather than experiencing symptomatic relief, however, the patient experienced paradoxical worsening of her clinical manifestations at a more rapid rate, and she was referred to our clinic for evaluation after 3 months.

The patient's neurological examination on presentation revealed intact visual acuity, partial ptosis, hypertropia, and constricted pupils on the left side, suggestive of Horner syndrome and a left trochlear nerve palsy. Movements pertaining to the oculomotor and abducens nerves were intact. Other findings on systemic examination were unremarkable. Repeat imaging demonstrated rapid growth of her left cavernous lesion, which now measured $10 \times 12 \times$ $19 \mathrm{~mm}$ (Fig. 1B). A plan was developed to proceed with tissue sampling to obtain a histological diagnosis.

\section{Minimally Invasive Cavernous Sinus Biopsy}

To rule out leukemic deposits, we performed a minimally invasive LOET approach using stereotactic neuronavigation for biopsy of the lesion (Video 1).

VIDEO 1. Illustrative video of the surgical steps for a lateral orbi-

totomy extradural transcavernous (LOET) approach. Copyright

Department of Neurosurgery, University of Utah. Published with permission. Click here to view.

A small, Y-shaped incision was made in the lateral can-
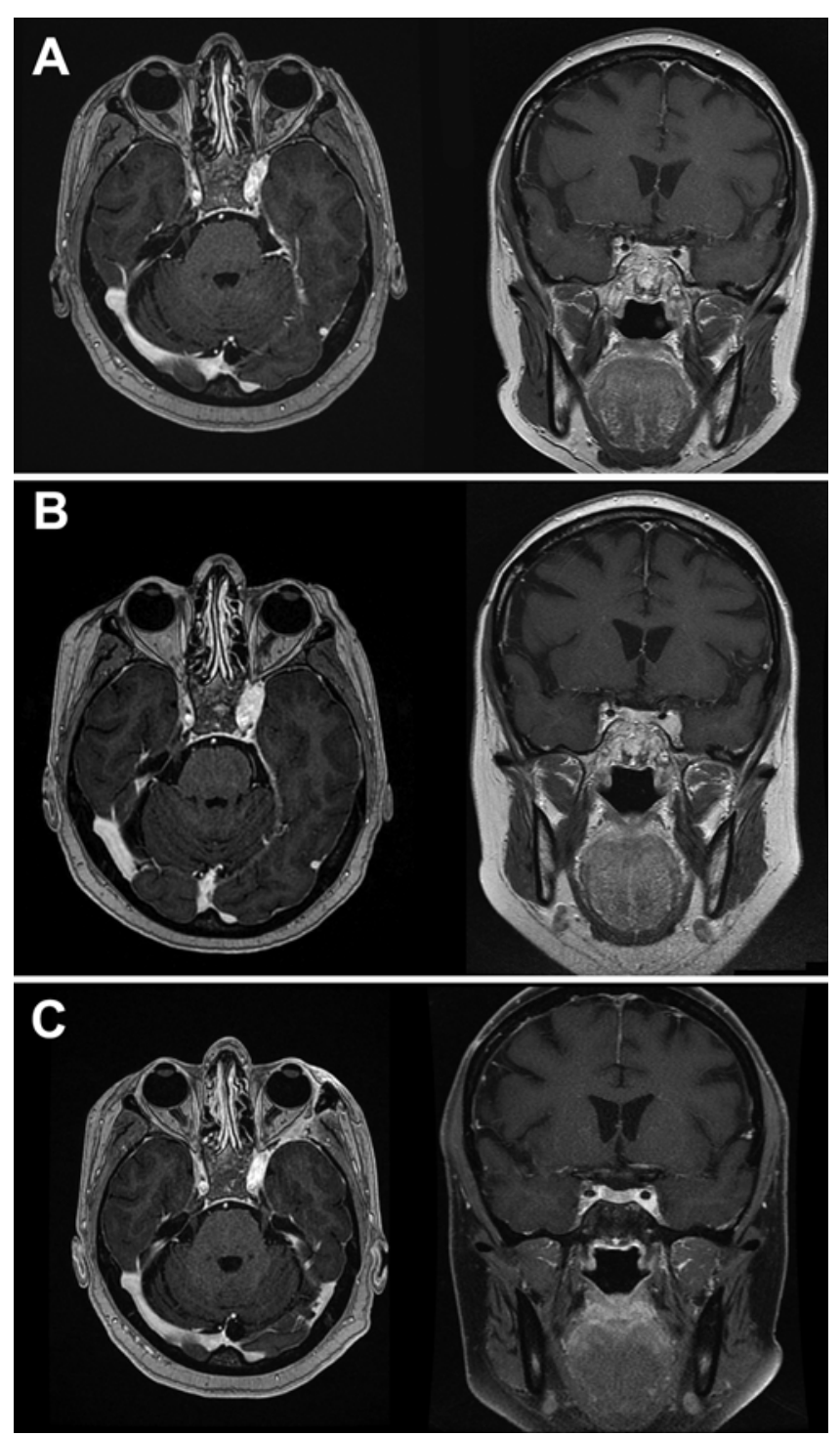

FIG. 1. A: Gadolinium-enhanced axial (left) and coronal (right) MR images of the brain revealing a $6 \times 8 \times 12-\mathrm{mm} \mathrm{T1/T2} \mathrm{isointense,} \mathrm{homo-}$ geneously enhancing lesion of the left cavernous sinus, with its predominant bulk anterolateral to the ICA. B: Repeat gadolinium-enhanced MR images of the brain obtained 3 months later demonstrating rapid growth of the left cavernous lesion, which now measured $10 \times 12 \times 19 \mathrm{~mm}$.

C: Follow-up gadolinium-enhanced MR images of the brain demonstrating partial regression of the left cavernous sinus tumor, correlating with symptomatic improvement.

thus of the left eye. Using subperiosteal dissection, we exposed approximately 1 inch of lateral orbital bone. A small lateral orbitotomy of about $2 \mathrm{~cm}$ was then performed using high-speed drills. We completed further drilling of the lateral orbital wall and deeper sphenoid wing under neuronavigation guidance. Under direct vision, the outer layer of cavernous sinus was peeled away extradurally to expose the tumor bulk, which was partly decompressed, and a specimen was sent for histological evaluation. The biopsied tumor tissue was found to be infiltrative, grayish, firm, and moderately vascular. After hemostasis was achieved, the lateral orbital rim was reconstructed using a 

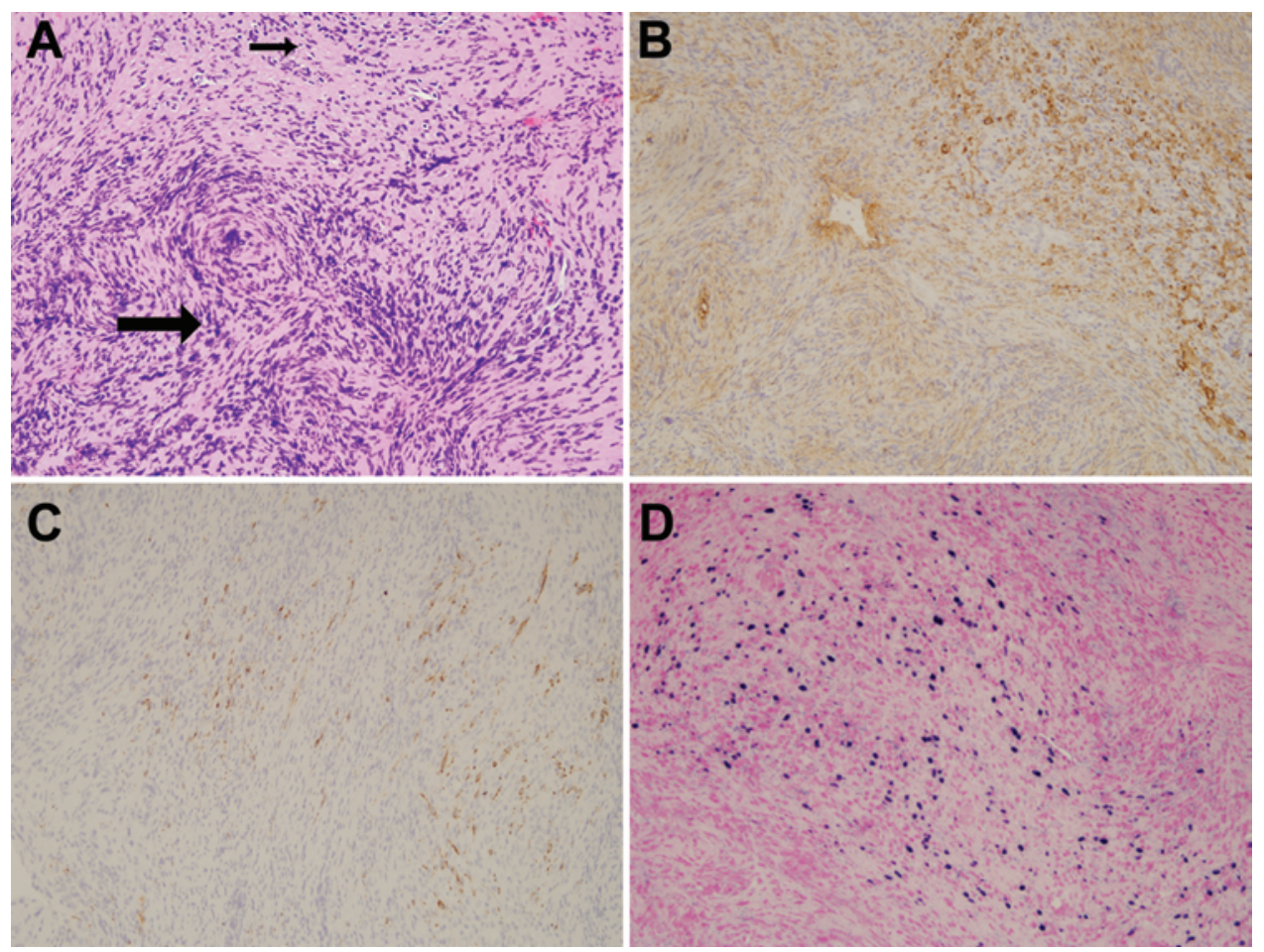

FIG. 2. Photomicrographs of the EBV-associated SMT showing short intersecting fascicles of eosinophilic spindled cells with tapered nuclei (thick arrow) and inflammatory infiltrate (thin arrow) (A, H \& E), spindle cells with membranous and cytoplasmic positivity for SMA (B), spindle cells with focal membranous and cytoplasmic positivity for desmin (C), and spindle cells with strong nuclear staining for EBV (D, EBV in situ hybridization). Original magnification $\times 200$. Figure is available in color online only.

thin and low-profile miniplate and screws. The wound was closed in layers using subcuticular suturing techniques.

\section{Histopathological Examination}

Histological evaluation showed a spindle cell lesion in an extensive background of reactive lymphoid cells. The lesion was composed of elongated cells, grouped in short, interlacing fascicles. The nuclei were tapered with occasional prominent nucleoli. A brisk inflammatory infiltrate including prominent histiocytes was noted. No necrosis or mitoses were identified. Given the patient's clinical history of acute myeloid leukemia, a battery of immunohistochemical stains was performed to exclude an intraaxial presentation of a hematolymphoid neoplasm. These stains revealed a polytypic infiltrate of largely histiocytes with no evidence of recurrent acute myeloid leukemia. Immunohistochemical evaluation of the spindle cells with smooth muscle actin (SMA) and desmin showed moderate and focal positivity, respectively, in the smooth muscle cells. Additionally, EBV-encoded RNA in situ hybridization showed diffuse positivity in the spindled tumor cells (Fig. 2). Hence, the diagnosis of EBV-associated SMT was made. The proliferative index, designated by a Ki 67 immunohistochemical stain, showed moderate-tohigh staining.

\section{Postoperative Course}

The patient's steroids were discontinued, and she was discharged the next day for an uneventful recovery. She had an acceptable cosmetic outcome for her lateral canthal incision. At her last follow-up visit, 3 months after surgery, the patient was doing well with partial relief of her symptoms, without any adjuvant chemotherapy, radiotherapy, or antiviral therapy. Her follow-up scan revealed partial regression of the tumor, correlating with symptomatic improvement (Fig. 1C).

\section{Discussion}

The differential diagnosis of EBV-associated SMTs includes primary central nervous system leiomyosarcoma, meningioma, hemangiopericytoma/solitary fibrous tumors, inflammatory myofibroblastic tumor, and sarcomatous differentiation of primary central nervous system tumors such as glioblastoma. ${ }^{16,18}$ The diagnosis is elucidated using immunohistochemical stains including smooth muscle markers (SMA, desmin, H-caldesmon) and importantly, in situ hybridization for EBV.

\section{EBV-Associated PT-SMT Pathogenesis and Detection}

Histologically, EBV-associated SMTs consist of short interlacing fascicles of relatively well-differentiated spindled, smooth muscle cells. A lymphocytic infiltrate is commonly present. ${ }^{5}$ At least one smooth muscle marker, including SMA, caldesmon, and desmin stains, is positive. ${ }^{5}$ By definition, EBV must be identified in the smooth muscle cells. All commonly described features were identified in this case. Historically, an effort had been made to classify EBV-associated smooth muscle lesions into benign and malignant categories (i.e., leiomyoma or leio- 
myosarcoma) using histological features such as cellularity, cytological atypia, necrosis, and hemorrhage; however, these features have not been definitively linked with prognosis. ${ }^{16}$ Mitotic activity either by mitotic count per high power field or proliferative index (Ki 67) has been shown to be the most important histological predictive factor. ${ }^{16}$

As per the initial report by McClain et al., ${ }^{13}$ EBV receptor CD21 may be a prerequisite for EBV infection of smooth muscle cell. Since smooth muscle cells do not express CD21 EBV surface receptor, some authors suggest the potential role of cell fusion with EBV-infected lymphocytes as a route for virus entry into nonlymphoid cells. ${ }^{19}$ In a majority of EBV-associated SMTs, viral infection generally precedes malignant/sarcomatous transformation..$^{13}$ Early literature emphasized EBV seropositivity as a key surrogate for past infection and consequent development of these tumors $;^{13}$ however, authors of recent publications have observed that seropositivity alone may not be sufficient to ascertain viral association of SMT, since it can simply be an epiphenomenon of immunosuppression. ${ }^{6,7}$ More precise methods for diagnosing EBV genetic material to confirm its association in tumors include PCR for EBV-DNA, immunohistochemistry for Latent membrane protein-1 (LMP-1), and EBV-encoded small RNA (EBER) ${ }^{6-8}$ EBER in situ hybridization diagnoses $98 \%$ of cases of PT-SMT?

PT-SMTs have an activated phosphoinositide-3-kinase (PI-3 K)/mammalian target of rapamycin (mTOR) signaling pathway. ${ }^{14}$ In addition, there is increased expression of v-myc avian myelocytomatosis viral oncogene homo$\log$ (MYC) in its pathogenesis. ${ }^{9}$ The tumor progenitor cell is believed to arise from an aberrant myogenous vascular wall cell., ${ }^{79}$ The fact that the majority of intracranial SMTs arise near cerebral veins and dural venous sinuses supports this hypothesis, although the exact site of origin is still a mystery. ${ }^{7,9}$

\section{Natural History of EBV-Associated PT-SMT}

EBV-associated SMTs can present as solitary or multifocal lesions. Because of their rarity and the lack of a national registry, their exact incidence is not known, but they are presumed to occur in less than $1 \%$ of the immunocompromised population.7 PT-SMT has a predisposition for involvement of the liver, followed by the lungs, larynx, pharynx, gut, spleen, and rarely the central nervous system. ${ }^{7,9}$ The average latency period after organ transplantation before the onset of these tumors is 4 years; early-onset tumors ( $<1$ year) comprise just $4 \%$ of reported cases. ${ }^{9}$ The majority of PT-SMTs arise after kidney transplantation $(60 \%)$, and the most common site of involvement is the liver (56\%). ${ }^{9}$ Hematopoietic stem cell transplantation-associated SMTs (extracranial) have been reported in 3 cases, but none of the previous cases had intracranial involvement. ${ }^{9}$ The average time interval before manifestation of symptoms from PT-SMT was 74 months. ${ }^{9}$ Our patient had a much faster progression of her clinical disease, with only a 24-month interval between peripheral blood stem cell transplantation and onset of clinical symptoms from PT-SMT, suggestive of a much more aggressive natural history. Her condition was partly aggravated by multiple relapses followed by multiple cycles of chemotherapy and later by consumption of oral steroids, both factors contributing to potential recurrent bouts of EBV viremia. Despite the multifocal and multicentric nature of SMT disease, these lesions have a very low propensity to metastasize and generally have a more benign clinical course than their non-EBV-induced congeners. ${ }^{7,9}$ Intracranial involvement is associated with worse prognosis, however, as approximately one-third of patients die within 6 months of symptom onset. ${ }^{9}$

\section{Treatment Options, Prognosis, and Outcome}

Therapeutic strategy in these cases depends not only on the tumor localization and potential resectability but also on the history of immunosuppression or immunodeficiency. ${ }^{5,7,9,16}$ Surgical debulking can be used in addition to adjustment of immunosuppression, although the outcomes are similar. ${ }^{5,7,16}$ There is no specific therapeutic advantage for adjuvant chemotherapy, radiotherapy, or antiviral therapy. ${ }^{5,7,9,16}$ Newer potential treatment options include mTOR/AKT inhibitors like sirolimus and EBV-specific cytotoxic T-cell therapies. ${ }^{2,7,9,20}$ Survival analysis does not show any differences in overall prognosis related to modality of treatment, occurrence of multiple tumors, tumor size, or sarcoma-like histological features, ${ }^{7}$ but as previously mentioned, intracranial involvement indicates poor prognosis for patients with PT-SMT?

\section{Lateral Orbitotomy Extradural Transcavernous Approach}

The cavernous sinus is aptly described as an "anatomical jewel box," in part because it harbors lesions of diverse origin. Despite advances in neuroimaging, conclusive diagnosis of certain cryptic cavernous sinus lesions is challenging without tissue sampling, yet surgical access to the cavernous sinus is a formidable task. The use of the minimally invasive LOET approach developed by the senior author (W.T.C.) offers a safe alternative to access this region. Drilling of the lateral orbital wall and deeper sphenoid wing exploration under neuronavigation guidance helps in precise localization of the cavernous sinus lesion, which is particularly important for such tumors located lateral to the intracavernous ICA. This LOET approach offers a practical, reliable, and low-risk minimally invasive technique for cavernous sinus biopsy. The advantages of this approach over its predecessors are the small skin incision, minimal soft-tissue dissection and blood loss, shorter incision-to-target distance, minimal brain retraction, extradural approach with direct trajectory to the anterolateral cavernous sinus, adequate tissue sampling under direct vision, and reduced hospital stay.

\section{Conclusions}

A high index of clinical suspicion is necessary to identify cryptic intracranial lesions in high-risk immunocompromised patients after peripheral blood stem cell transplantation and multiple cycles of chemotherapy. EBVassociated SMTs can have a presentation similar to THS, but the paradoxical response to steroids with symptomatic worsening should suggest underlying EBV-associated neoplasia in immunosuppressed patients. Intracranial involvement of PT-SMT is typically along dural venous 
sinuses, cerebral veins, and dura mater and is associated with worse prognosis. The LOET technique offers a minimally invasive approach to access cavernous sinus lesions under direct vision with a shorter trajectory. EBER in situ hybridization is the confirmatory tool to diagnose this rare entity. Treatment for PT-SMTs includes adjustment of immunosuppression and reasonable tumor debulking if feasible. Adjuvant therapies have a minimal role in overall management of these lesions.

\section{Acknowledgments}

We thank Kristin Kraus, MSc, our medical editor, for her contribution to manuscript editing and Vance Mortimer, our video editor, for his contribution to editing the operative video.

\section{References}

1. Altay T, Patel BC, Couldwell WT: Lateral orbital wall approach to the cavernous sinus. J Neurosurg 116:755-763, 2012

2. Belingheri M, Comoli P, Locatelli F, Baldanti F, Martina V, Giani M, et al: Successful medical treatment of EBV smooth muscle tumor in a renal transplant recipient. Pediatr Transplant 14:E101-E104, 2010

3. Boudjemaa S, Boman F, Guigonis V, Boccon-Gibod L: Brain involvement in multicentric Epstein-Barr virus-associated smooth muscle tumours in a child after kidney transplantation. Virchows Arch 444:387-391, 2004

4. Chaves NJ, Kotsimbos TC, Warren MA, McLean CA, Spelman DW, Williams TJ, et al: Cranial leiomyosarcoma in an Epstein-Barr virus (EBV)-mismatched lung transplant recipient. J Heart Lung Transplant 26:753-755, 2007

5. Deyrup AT, Lee VK, Hill CE, Cheuk W, Toh HC, Kesavan S, et al: Epstein-Barr virus-associated smooth muscle tumors are distinctive mesenchymal tumors reflecting multiple infection events: a clinicopathologic and molecular analysis of 29 tumors from 19 patients. Am J Surg Pathol 30:75-82, 2006

6. Hussein K, Maecker-Kolhoff B, Klein C, Kreipe H: [Transplant-associated lymphoproliferation.] Pathologe 32:152158, 2011 (Ger)

7. Hussein K, Rath B, Ludewig B, Kreipe H, Jonigk D: Clinicopathological characteristics of different types of immunodeficiency-associated smooth muscle tumours. Eur J Cancer 50:2417-2424, 2014

8. Jonigk D, Izykowski N, Maegel L, Schormann E, MaeckerKolhoff B, Laenger F, et al: MicroRNA expression in Epstein-Barr virus-associated post-transplant smooth muscle tumours is related to leiomyomatous phenotype. Clin Sarcoma Res 3:9, 2013

9. Jonigk D, Laenger F, Maegel L, Izykowski N, Rische J, Tiede C, et al: Molecular and clinicopathological analysis of Epstein-Barr virus-associated posttransplant smooth muscle tumors. Am J Transplant 12:1908-1917, 2012

10. Kaphan E, Eusebio A, Witjas T, Donnet A, Vacher-Coponat $\mathrm{H}$, Figarella-Branger D, et al: [Primary leiomyosarcoma of the cavernous sinus associated with Epstein-Barr virus in a kidney graft.] Rev Neurol (Paris) 159:1055-1059, 2003 (Fr)

11. Kazmi SA, Aizenberg MR, Harper JL, McComb RD: Multifocal histologically malignant Epstein-Barr virus-associated smooth muscle tumor in a pediatric transplant patient with an indolent course. Int J Surg Pathol 22:186-189, 2014

12. Lee ES, Locker J, Nalesnik M, Reyes J, Jaffe R, Alashari M, et al: The association of Epstein-Barr virus with smooth- muscle tumors occurring after organ transplantation. N Engl J Med 332:19-25, 1995

13. McClain KL, Leach CT, Jenson HB, Joshi VV, Pollock BH, Parmley RT, et al: Association of Epstein-Barr virus with leiomyosarcomas in children with AIDS. N Engl J Med 332:12-18, 1995

14. Ong KW, Teo M, Lee V, Ong D, Lee A, Tan CS, et al: Expression of EBV latent antigens, mammalian target of rapamycin, and tumor suppression genes in EBV-positive smooth muscle tumors: clinical and therapeutic implications. Clin Cancer Res 15:5350-5358, 2009

15. Pritzker KP, Huang SN, Marshall KG: Malignant tumours following immunosuppressive therapy. Can Med Assoc J 103:1362-1365, 1970

16. Purgina B, Rao UN, Miettinen M, Pantanowitz L: AIDSrelated EBV-associated smooth muscle tumors: a review of 64 published cases. Pathol Res Int 2011:561548, 2011

17. Tahri A, Noel G, Figuerella-Branger D, Goncalves A, Feuvret L, Jauffret E, et al: [Epstein-Barr virus associated central nervous system leiomyosarcoma occurring after renal transplantation: case report and review of the literature.] Cancer Radiother 7:308-313, 2003 (Fr)

18. Takei H, Powell S, Rivera A: Concurrent occurrence of primary intracranial Epstein-Barr virus-associated leiomyosarcoma and Hodgkin lymphoma in a young adult. J Neurosurg 119:499-503, 2013

19. Timens W, Boes A, Vos H, Poppema S: Tissue distribution of the $\mathrm{C} 3 \mathrm{~d} / \mathrm{EBV}$-receptor: CD21 monoclonal antibodies reactive with a variety of epithelial cells, medullary thymocytes, and peripheral T-cells. Histochemistry 95:605-611, 1991

20. Toh HC, Teo M, Ong KW, Lee V, Chan E, Lee AS, et al: Use of sirolimus for Epstein-Barr virus-positive smooth-muscle tumour. Lancet Oncol 7:955-957, 2006

21. Zevgaridis D, Tsonidis C, Kapranos N, Venizelos I, Tsitsopoulos P, Tsitsopoulos P: Epstein-Barr virus associated primary intracranial leiomyoma in organ transplant recipient: case report and review of the literature. Acta Neurochir (Wien) 151:1705-1709, 2009

\section{Disclosures}

The authors report no conflict of interest concerning the materials or methods used in this study or the findings specified in this paper.

\section{Author Contributions}

Conception and design: Raheja. Acquisition of data: Raheja, Sowder. Analysis and interpretation of data: Sowder. Drafting the article: Raheja. Critically revising the article: all authors. Reviewed submitted version of manuscript: Couldwell, Raheja, Palmer, Rodriguez. Approved the final version of the manuscript on behalf of all authors: Couldwell. Video preparation: Couldwell.

\section{Supplemental Information \\ Videos}

Video 1. https://vimeo.com/165307365.

\section{Correspondence}

William T. Couldwell, Department of Neurosurgery, Clinical Neurosciences Center, University of Utah, $175 \mathrm{~N}$ Medical Dr. East, Salt Lake City, UT 84132. email: neuropub@hsc.utah.edu. 